\title{
Diferencias de género en las reacciones psicosomáticas de los estudiantes afectados por el estrés de los exámenes
}

\author{
Joanna Kosmala-Anderson, Louise M. Wallace
}

Health \& Lifestyles Interventions Research Centre, Universidad de Coventry

\section{Reino Unido}

j.kosmala-anderson@coventry.ac.uk 


\section{Resumen}

Introducción. Este estudio investiga la ansiedad y las estrategias de control emocional preexamen, como posibles mediadores de diferencias de género en la intensidad y tipo de reacciones psicosomáticas ante el estrés de exámenes, ambos auto-informados.

Método. La muestra consiste en 150 varones y 150 mujeres, estudiantes del último curso de secundaria o de universidad, que participaron voluntariamente en la encuesta. Los cuestionarios medían la intensidad y el tipo de reacciones psicosomáticas ante el estrés de los exámenes (Psychosomatic Reaction Inventory), el nivel de ansiedad pre-examen (State-Trait Anxiety Inventory) y la aplicación de estrategias de auto-control emocional (An Emotional SelfControl Strategies Questionnaire).

Resultados. El nivel de ansiedad pre-examen está correlacionado positivamente con la intensidad auto-informada de las reacciones psicosomáticas ante el estrés de los exámenes $(\mathrm{F}=34,23 ; \mathrm{gl}=2 ; \mathrm{p}<.001)$. Las estudiantes experimentaban un nivel más alto de ansiedad preexamen, reacciones más fuertes al estrés por exámenes $(\mathrm{t}=6.24 ; \mathrm{gl}=298 ; \mathrm{p}<.001)$, así como una mayor diversidad en estas reacciones. La alta ansiedad pre-examen de las mujeres se mostró como factor que mediaba su experiencia de las reacciones psicosomáticas más intensas en estrés de exámenes, $\mathrm{F}(2,297)=19,46 ; \mathrm{p}<.001)$. No existía ninguna influencia moderadora de la aplicación de ciertas estrategias de auto-control emocional por parte de cada género en la intensidad auto-informada de las reacciones psicosomáticas ante el estrés de los exámenes.

Palabras clave: género, estrés pre-examen, reacción psicosomática, estrategias de autocontrol emocional

\footnotetext{
Recepción del artículo: $\quad$ 02-04-2007

Aceptación provisional: $\quad 15-05-2007$

Aceptación definitiva: $\quad$ 20-08-2007
} 